\title{
Significant cytostatic effect of everolimus on a gefitinib-resistant anaplastic thyroid cancer cell line harboring PI3KCA gene mutation
}

\author{
NAOYOSHI ONODA ${ }^{1 *}$, MASANORI NAKAMURA $^{1 *}$, NAOKI AOMATSU $^{1}$, SATORU NODA $^{1}$, \\ SHINICHIRO KASHIWAGI ${ }^{1}$, KENTO KURATA ${ }^{1}$, SHINYA UCHINO ${ }^{2}$ and KOSEI HIRAKAWA ${ }^{1}$ \\ ${ }^{1}$ Department of Surgical Oncology, Osaka City University Graduate School of Medicine, Osaka, Osaka 545-8585; \\ ${ }^{2}$ Noguchi Thyroid Clinic, Beppu, Oita 874-0932, Japan
}

Received October 6, 2014; Accepted January 14, 2015

DOI: $10.3892 / \mathrm{mco} .2015 .496$

\begin{abstract}
We previously demonstrated the efficacy of gefitinib, a tyrosine kinase inhibitor of the epidermal growth factor receptor (EGFR), on an anaplastic thyroid cancer (ATC) cell line. We also observed that gefitinib was not effective in regulating cell growth in a different ATC cell line that exhibited an altered EGFR-initiated signal transduction pathway. In the present study, we attempted to regulate the downstream effector of EGFR-Akt-mammalian target of rapamycin (mTOR) pathway by an mTOR inhibitor, everolimus. A total of 8 ATC cell lines were employed, 7 of which were established in our institute. OCUT-2 was known to carry a mutation in the phosphoinositide-3-kinase, catalytic, $\alpha$ polypeptide gene (PI3KCA) and to be gefitinib-resistant, whereas ACT-1 exhibited a remarkable growth arrest by gefitinib. All the cell lines were tested for the cytotoxic effect of everolimus. The mechanisms of cellular toxicity were investigated by EGFR stimulation, cell cycle and concurrent exposure to paclitaxel. In OCUT-2, but not in any of the other cell lines, everolimus achieved a significant growth inhibition (inhibition of 30 and $50 \%$ was achieved by concentrations of 0.8 and $5 \mathrm{nM}$, respectively). The growth in OCUT-2 was inhibited by everolimus, even with concordant EGFR stimulation. This effect was demonstrated by a G2M cell cycle arrest. An additive effect of everolimus onto the cytotoxic effect of paclitaxel was demonstrated at a dose of 1-2 $\mathrm{nM}$. A significant growth inhibitory effect of everolimus on the gefitinib-resistant ATC cell line was demonstrated, suggesting a possible correlation between the efficacy of everolimus and PI3KCA gene mutation and the
\end{abstract}

Correspondence to: Dr Naoyoshi Onoda, Department of Surgical Oncology, Osaka City University Graduate School of Medicine, 1-4-3 Asahi-machi, Abeno-ku, Osaka, Osaka 545-8585, Japan

E-mail: nonoda@med.osaka-cu.ac.jp

*Contributed equally

Key words: anaplastic thyroid cancer, cell lines, mammalian target of rapamycin inhibitor, everolimus, resistance significance of molecular-targeted therapy in the management of ATC.

\section{Introduction}

Anaplastic thyroid cancer (ATC) is a rare entity, accounting for $1-3 \%$ of all thyroid cancer cases. ATC is one of the most aggressive malignancies in humans. Multimodal therapies, including surgery, radiation and chemotherapy, are generally used to manage this highly malignant disease. However, the prognosis is poor and the majority of the patients succumb to the disease within a year, with a median survival time of $<6$ months from the initial diagnosis (1-3). Recently, new approaches to treating ATC by targeted molecular markers have been developed to overcome therapeutic resistance (4-6). We previously demonstrated the efficacy of gefitinib, a tyrosine kinase inhibitor (TKI) of the epidermal growth factor receptor (EGFR), on an ATC cell line, as a potential novel therapeutic strategy. However, we observed that gefitinib was not effective in regulating cell growth in a different cell line that exhibited an altered EGFR-initiated signal transduction pathway (7). A mutation of the phosphoinositide-3-kinase, catalytic, $\alpha$ polypeptide gene (PI3KCA) was later identified in the gefitinib-resistant cell line (8). In the present study, we attempted to regulate the downstream effector of EGFR-Akt-mammalian target of rapamycin (mTOR) pathway by an mTOR inhibitor, everolimus, in a gefitinib-resistant cell line and to demonstrate the mechanism to overcome resistance to EGFR-targeted therapy.

\section{Materials and methods}

Chemicals. Everolimus (RAD001) was provided by Novartis (Basel, Switzerland). Paclitaxel was purchased from Wako Pure Chemical Industries, Ltd. (Osaka, Japan).

Cell lines and cell cultures. We used a panel of 8 authentic human ATC cell lines, including 2 cell lines (OCUT-2 and ACT-1) that were used in our previous experiment (7). OCUT-1-6 were established and characterized in our laboratory. A mutation of B-Raf V600E was found in OCUT-1-5 and a N-Ras mutation was found in the OCUT-6 and ACT-1 cell lines (9). OCUT-2 
Table I. Inhibitory concentrations (30 and 50\%) of everolimus in different cell lines.

\begin{tabular}{lccc}
\hline Cell line & Known gene mutations & $\mathrm{IC}_{30}(\mathrm{nM})$ & $\mathrm{IC}_{50}(\mathrm{nM})$ \\
\hline OCUT-1C & B-Raf & 14 & $-^{\mathrm{a}}$ \\
OCUT-1F & B-Raf & 8.5 & 80 \\
OCUT-2 & B-Raf, PI3KCA and EGFR & 0.8 & 5 \\
OCUT-3 & B-Raf & 2.5 & 19 \\
OCUT-4 & B-Raf & 8.4 & $-^{\mathrm{a}}$ \\
OCUT-5 & B-Raf & 14 & 24 \\
OCUT-6 & N-Ras & 17 & - $^{\mathrm{a}}$ \\
ACT-1 & N-Ras & 80 \\
\hline
\end{tabular}

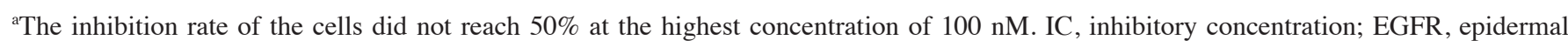
growth factor receptor.; PI3KCA, phosphoinositide-3-kinase, catalytic, $\alpha$ polypeptide gene.

was known to carry a mutation of the PI3CA gene (8). ACT-1 was kindly provided by Dr S. Ohata of Tokushima University. Each cell line was cultured in Dulbecco's modified Eagle's medium (DMEM) supplemented with $10 \%$ fetal bovine serum (FBS), $100 \mathrm{IU} / \mathrm{ml}$ penicillin and $100 \mathrm{mg} / \mathrm{ml}$ streptomycin at $37^{\circ} \mathrm{C}$ with $5 \% \mathrm{CO}_{2}$ in a humidified incubator.

MTT assay. The inhibitory effects of everolimus on the viability of these cell lines were measured by the MTT assay (7). Cells $\left(1 \times 10^{3}\right)$ were seeded in each well of a 96 -well plastic culture plate and left overnight under the same conditions. The cells were then treated with the intended doses of everolimus for 3 days. After the incubation period, MTT was added to a final concentration of $0.5 \mathrm{mg} / \mathrm{ml}$ and the cells were incubated again for $2 \mathrm{~h}$ under the same conditions. The culture plate was centrifuged at $200 \mathrm{x} \mathrm{g}$ for $5 \mathrm{~min}$ and the supernatant was removed. Dimethyl sulfoxide was added for reaction and the absorbency was measured with a Model 550 microplate reader (Bio-Rad Laboratories, Hercules, CA, USA) and calculated using the supplied software. The experiments were performed three times independently, in triplicate each time and the average values of the three independent experiments were calculated.

The effect of everolimus on cell viability following paclitaxel treatment was also measured by the MTT assay. OCUT-2 cells were exposed to $1 \mathrm{nM}$ of everolimus $1 \mathrm{~h}$ before, concomitantly, or $1 \mathrm{~h}$ after treatment with 1-100 $\mathrm{nM}$ of paclitaxel for $72 \mathrm{~h}$.

Effect of everolimus on tumor cell proliferation under EGF stimulation in vitro. Cells $\left(5 \times 10^{4}\right)$ were spread onto a $10-\mathrm{mm}$ plastic dish and left overnight. The cells were then cultured in DMEM without FBS. One nmol of EGF (no. 26190U, Upstate, Lake Placid, NY, USA) was added to a plate to stimulate the EGFR of the cells. The efficacy of everolimus (1 and $10 \mathrm{nM})$ was investigated by immediate addition following EGF exposure. The cells were counted after $48 \mathrm{~h}$ of incubation. The experiments were performed independently in triplicate.

Cell cycle analysis by flow cytometry. Flow cytometry was used to measure the DNA content of individual cells, which allowed us to assess the cell-cycle profiles of the cells treated with everolimus. In preparation for flow cytometry, cells treated with $2 \mathrm{nM}$ of everolimus for $16-72 \mathrm{~h}$ were collected

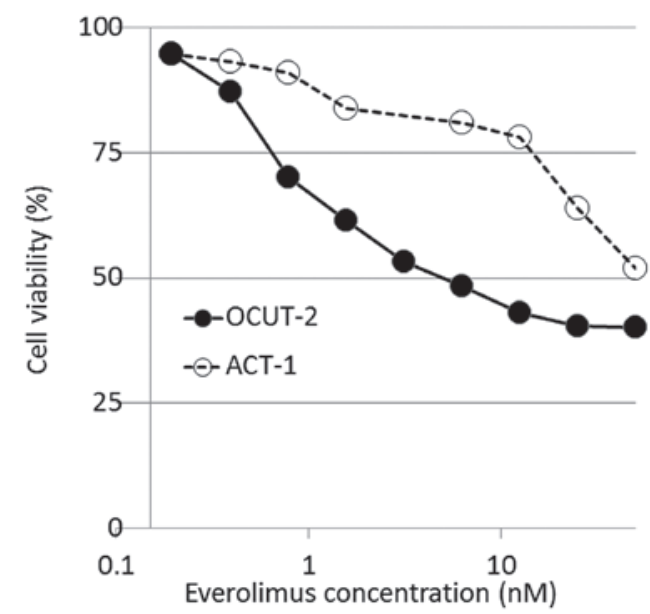

Figure 1. Results of the MTT assay. Similar to the results of the ACT-1 cell line, everolimus exerted a weak growth inhibitory effect on all the cell lines, apart from the gefitinib-resistant OCUT-2 cell line, which harbors a PI3KCA gene mutation and in which everolimus achieved a significant growth inhibition. PI3KCA, phosphoinositide-3-kinase, catalytic, $\alpha$ polypeptide gene.

following brief trypsinization, washed with phosphate-buffered saline and fixed with $70 \%$ cold ethanol. The samples were then treated with ribonuclease (R6513; Sigma-Aldrich Corp., St. Louis, MO, USA), stained with $10 \mathrm{mg} / \mathrm{ml}$ propidium iodide and analyzed by a FACScan cell sorter (Becton Dickinson, Mountain View, CA, USA). Cell cycle distributions were quantified using CellQuest software (Becton Dickinson).

\section{Results}

Growth inhibitory effect of everolimus. A similar growth inhibitory effect of everolimus was observed in all the cell lines, except for OCUT-2. The 30 and 50\% inhibitory concentrations of everolimus ranged from 8.4 to 23.5 and from 19 to $>100 \mathrm{nM}$, respectively (Table I). However, in OCUT-2, everolimus achieved a significant growth inhibition compared to that in other cell lines, with 30 and $50 \%$ inhibitory concentrations of 0.8 and $5 \mathrm{nM}$, respectively. The maximal growth inhibitory effect of everolimus on OCUT-2 cells was demonstrated at the concentration of $\sim 20 \mathrm{nM}$, where $\sim 60 \%$ of the cells were growth-inhibited and no further effect was observed 


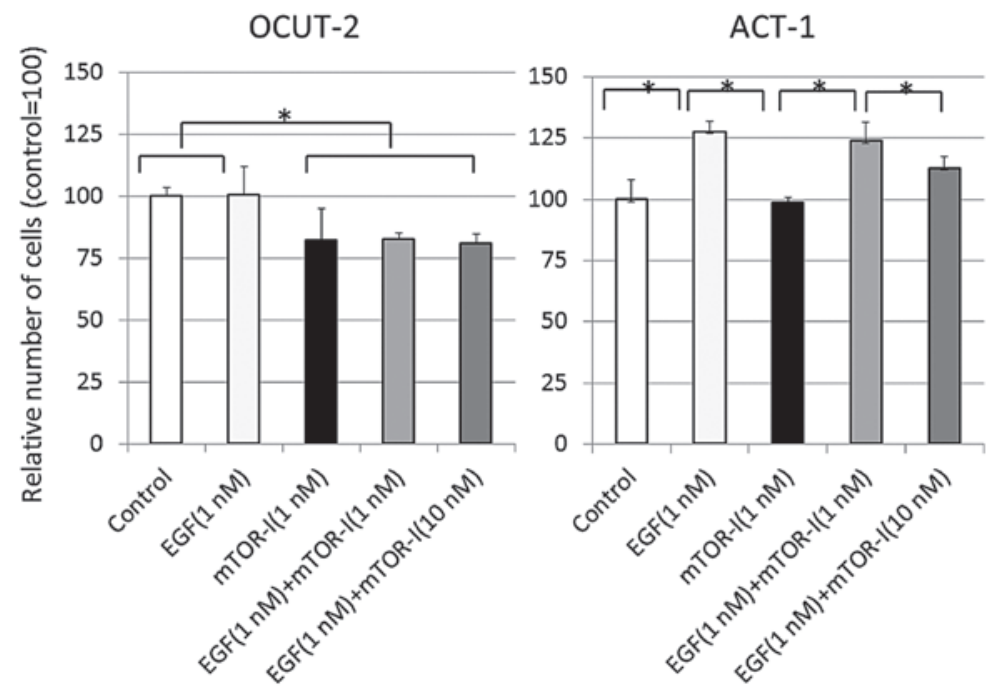

Figure 2. Cancer cell proliferation in reaction to everolimus under epidermal growth factor (EGF) stimulation. OCUT-2 cells did not exhibit any change in proliferation under EGF stimulation and everolimus achieved a significant inhibition of cell proliferation, even under EGF stimulation. By contrast, ATC-1 cell proliferation was significantly stimulated by EGF, but not affected by everolimus. ("P<.01). mTOR, mammalian target of rapamycin.

\section{A}
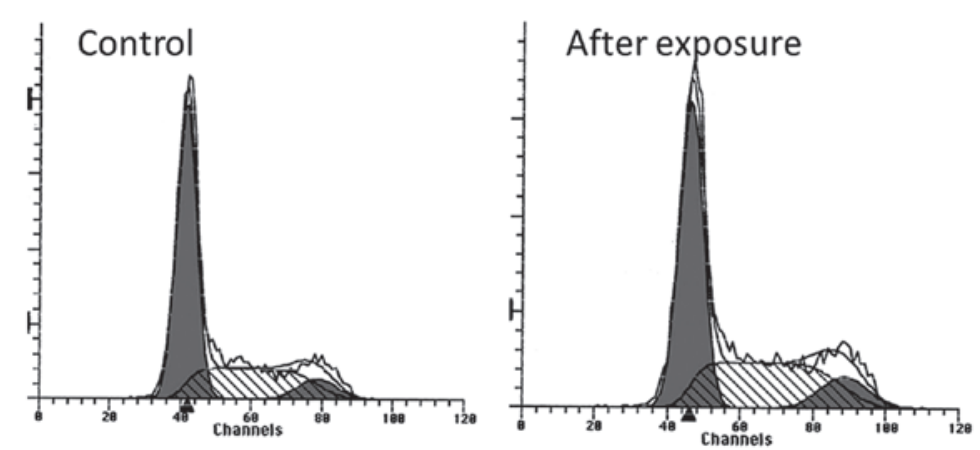

B

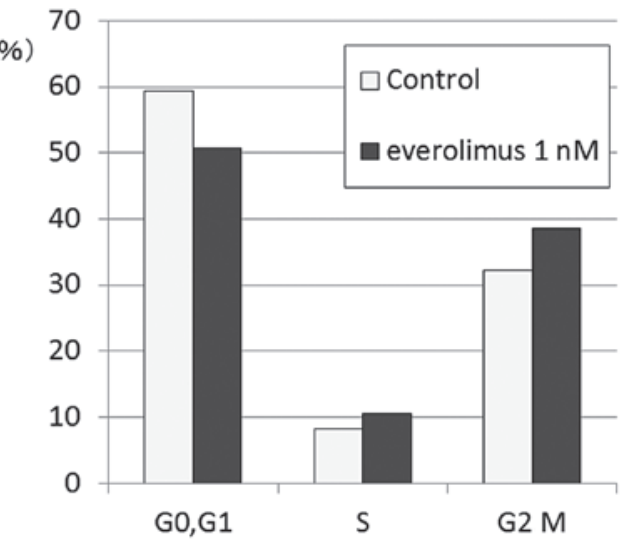

Figure 3. (A) DNA histograms of control cells and cells following exposure to everolimus. (B) An increase in S and G2M phase cell population was observed following exposure, indicating G2M cell cycle arrest.

by increasing the concentration (Fig. 1). There was a significant difference in the sensitivity to everolimus between the ACT-1 and OCUT-1, $-3,-4,-5$ and -6 cell lines (data not shown).

The proliferation of cancer cells in reaction to EGF clearly differed between the ACT-1 and OCUT- 2 cell lines. ACT- 1 cells exhibited a significant upregulation of proliferation by stimulation with $1 \mathrm{nM}$ of EGF, as previously reported (7). There was no change in OCUT-2 cell proliferation by EGF stimulation.
Everolimus achieved a significant inhibition of cell proliferation in the OCUT-2 cell line, even under EGF stimulation. A concentration of $1 \mathrm{nM}$ was sufficient to achieve maximal growth inhibition in OCUT-2 cells. By contrast, ATC-1 cell proliferation was significantly stimulated by EGF, but not affected by exposure to $1 \mathrm{nM}$ of everolimus. A high concentration $(10 \mathrm{nM})$ of everolimus was required to achieve a partial inhibition of the upregulated cellular growth of ACT-1 stimulated by EGF (Fig. 2). 


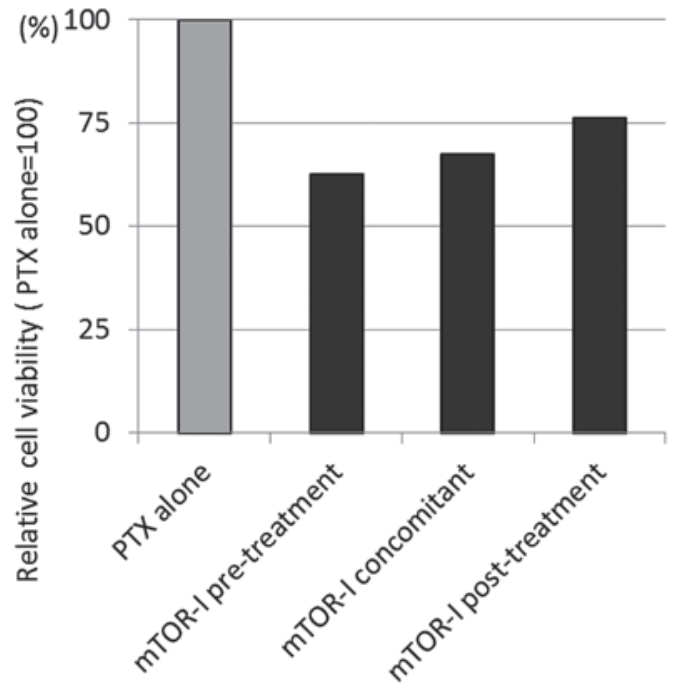

Figure 4. Additive effect of everolimus onto the cytotoxic effect of paclitaxel (PTX). The additive effect of everolimus was more pronounced when it was administrated prior to paclitaxel exposure. mTOR, mammalian target of rapamycin.

G2M cell cycle arrest was observed after a 16-h exposure to everolimus ( $1 \mathrm{nM})$, with a $19 \%$ increased ratio of cells in the G2M phase (32-39\%). The G2M arrest continued until $72 \mathrm{~h}$ after exposure (Fig. 3).

An additive effect of everolimus onto the cytotoxicity of paclitaxel was demonstrated at a dose of $1 \mathrm{nM}$. There was a $32 \%$ decrease in cell viability following exposure of OCUT-2 cells to $1 \mathrm{nM}$ of paclitaxel in the presence of $1 \mathrm{nM}$ of everolimus compared to paclitaxel alone. The additive effect of everolimus was more pronounced when it was administered prior to paclitaxel exposure (Fig. 4).

\section{Discussion}

Conventionally, ATC has been managed with a multimodal therapeutic approach by combining surgery, chemotherapy and external beam radiation. However, the effect of these approaches is limited due to the highly aggressive nature of the tumor and rapid acquisition of resistance to treatment. To overcome this resistance, molecular-targeted approaches have been applied as a possible novel therapeutic strategy. To date, there has been some success in managing this disease (4-6). EGFR is a well known cell membranous receptor and has been reported to be highly expressed in ATC cells (10). Thus, we attempted to manage the disease by inhibiting the kinase activity of EGFR by gefitinib in a previous study and demonstrated the significant efficacy of this TKI in inhibiting cancer cell growth. In the ACT-1 cell line, the cell-proliferating signal through EGFR to mitogen-activated protein kinase kinase (MEK) was clearly inhibited by gefinitib (7). Furthermore, this significant effect was not observed in OCUT-2, a cell line exhibiting a lower level of EGFR expression.

There are two major pathways inducing the proliferation of cancer cells downstream of cell surface EGFR, namely the Raf/Ras/MEK and PI3K/Akt/mTOR pathways. Alterations in both pathways have often been observed in thyroid cancer (11-15). B-Raf, Ras, PI3KCA and phosphatase and tensin homolog mutations have also been reported in ATC (16-19). Several cell lines harbor one of these gene mutations. By contrast, OCUT-2 is a unique cell line exhibiting alterations in both B-Raf and PI3KCA genes, resulting in completely aberrant proliferation signal generation independent of EGFR-mediated signaling (9). The results of our previous study may, thus, be interpreted as that the efficacy of EGFR-targeted therapy may be determined by the signaling status downstream of EGFR. Therefore, in this study, we attempted to inhibit mutated PI3KCA-generated aberrant growth signaling by an mTOR inhibitor to overcome gefitinib resistance.

A significantly more prominent inhibitory effect on cell proliferation was demonstrated by everolimus in the OCUT-2 cell line, compared to that in other cell lines not carrying alterations in the PI3K/Akt/mTOR pathway, as expected. This observation may indicate the significance of detecting alterations in the PI3K/Akt/mTOR signaling pathway as an indicator of the possible efficacy of everolimus. According to the results of the effects on ACT-1, everolimus was only able to impair part of the proliferation signal generated from EGFR when the signaling pathway was intact. By contrast, a major signal for proliferation was blocked in OCUT-2 cells harboring an aberrantly activated PI3K/Akt/mTOR pathway by a PI3KCA mutation. In addition, OCUT-2 also carrying an active B-Raf mutation, which should cause alternative generation of the proliferating signal in the absence of the PI3K/Akt/mTOR axis, resulted in the incomplete impairment of cell growth.

We observed a marginal increase in the number of cells in the $\mathrm{S}$ and $\mathrm{G} 2 \mathrm{M}$ phase following exposure to everolimus in the OCUT-2 cell line, suggesting G2M cell cycle arrest. Everolimus is known to cause G0G1 arrest by inhibiting the expression of cyclin D1. However, a very high concentration (5,000-20,000 $\mathrm{nM}$ ) of everolimus was reportedly required to achieve G0G1 arrest $(20,21)$ compared to the concentration we used in the present experiment. The concentration of everolimus we used in this study was $2 \mathrm{nM}$, since we were unable to achieve additional inhibition in cell proliferation when increasing the dose to $>20 \mathrm{nM}$ in the initial experiments investigating the effect of everolimus on cell viability. The DNA histogram analysis did not identify any cell population with lower or fragmented DNA contents, suggesting apoptosis. These observations may indicate that the effect of everolimus is expressed as a moderate cell cycle arrest in G2M, but not as cell killing within physiological doses.

In the present study, we demonstrated that everolimus inhibited cell growth in cancer cells harboring an altered $\mathrm{PI} 3 \mathrm{~K} / \mathrm{Akt} / \mathrm{mTOR}$ signaling pathway. This effect was limited to cell growth arrest and no complete cytotoxicity was observed. As stated earlier, alterations in the PI3K/Akt/mTOR pathway in ATC was not a major genetic abnormality, compared to B-Raf- or Ras-mediated pathway. Farstino et al (22) suggested the possible mechanism of LKB1-mediated mTOR pathway upregulation in thyroid carcinoma harboring the B-Raf mutation. Dual inhibition of the Raf/Ras/MEK and PI3K/Akt/mTOR pathways was attempted, with promising preclinical results (23). A combination of a cytotoxic drug with everolimus may be another practical choice to increase the efficacy of treatment. We observed an additive effect of paclitaxel to that of everolimus in the OCUT- 2 cell line. Paclitaxel exerts its cytotoxic effect by inhibiting the polymerization of tubulin 
in the G2M phase. Further studies are required to maximize the inhibitory effect of everolimus on the mTOR pathway.

In conclusion, a significant growth in hibitory effect of everolimus on a gefitinib-resistant ATC cell line was demonstrated. A possible correlation between the efficacy of everolimus and PI3KCA gene mutation requires further investigation using additional ATC samples with accurate information on genetic alterations. This study indicated the significance of identifying target molecules, or applying target-oriented therapeutic strategies in managing patients with highly malignant ATC.

\section{Acknowledgements}

This study was supported in part by a Grant-in-Aid for Scientific Research (Kakenhi no. 25461992).

\section{References}

1. Smallridge RC: Approach to the patient with anaplastic thyroid carcinoma. J Clin Endocrinol Metab 97: 2566-2572, 2012.

2. Akaishi J, Sugino K, Kitagawa W, et al: Prognostic factors and treatment outcomes of 100 cases of anaplastic thyroid carcinoma. Thyroid 21: 1183-1189, 2011.

3. Sugitani I, Miyauchi A, Sugino K, Okamoto T, Yoshida A and Suzuki S: Prognostic factors and treatment outcomes for anaplastic thyroid carcinoma: ATC research consortium of Japan cohort study of 677 patients. World J Surg 36: 1247-1254, 2012.

4. Cabanillas ME, Waguespack SG, Bronstein Y, Williams MD, Feng L, Hernandez M, Lopez A, Sherman SI and Busaidy NL: Treatment with tyrosine kinase inhibitors for patients with differentiated thyroid cancer: the M. D. Anderson experience. J Clin Endocrinol Metab 95: 2588-2595, 2010

5. Benvenga S: Emerging therapies in sight for the fight against dedifferentiated thyroid cancer. J Clin Endocrinol Metab 96: 347-350, 2011.

6. Rosove MH, Peddi PF and Glaspy JA: BRAF V600E inhibition in anaplastic thyroid cancer. N Engl J Med 368: 684-685, 2013.

7. Nobuhara Y, Onoda N, Yamashita Y, Yamasaki M, Ogisawa K, Takashima T, Ishikawa T and Hirakawa K: Efficacy of epidermal growth factor receptor-targeted molecular therapy in anaplastic thyroid cancer cell lines. Br J Cancer 92: 1110-1116, 2005.

8. Liu D, Hou P, Liu Z, Wu G and Xing M: Genetic alterations in the phosphoinositide 3-kinase/Akt signaling pathway confer sensitivity of thyroid cancer cells to therapeutic targeting of Akt and mammalian target of rapamycin. Cancer Res 69: 7311-7319, 2009.

9. Onoda N, Nakamura M, Aomatsu N, Noda S, Kashiwagi S and Hirakawa K: Establishment, characterization and comparison of seven authentic anaplastic thyroid cancer cell lines retaining clinical features of the original tumor. World J Surg 38: 688-695, 2014.
10. Wiseman SM, Masoudi H, Niblock P, Turbin D, Rajput A, Hay J, Bugis S, Filipenko D, Huntsman D and Gilks B: Anaplastic thyroid carcinoma: expression profile of targets for therapy offers new insights for disease treatment. Ann Surg Oncol 14: 719-729, 2007.

11. Rusinek D, Szpak-Ulczok S and Jarzab B: Gene expression profile of human thyroid cancer in relation to its mutational status. J Mol Endocrinol 47: R91-R103, 2011.

12. Xing M, Alzahrani AS, Carson KA, et al: Association between BRAF V600E mutation and mortality in patients with papillary thyroid cancer. JAMA: 309: 1493-1501, 2013.

13. Kim TH, Park YJ, Lim JA, et al: The association of the BRAF(V600E) mutation with prognostic factors and poor clinical outcome in papillary thyroid cancer: a meta-analysis. Cancer 118: 1764-1773, 2012.

14. Garcia-Rostan G, Zhao H, Camp RL, Pollan M, Herrero A, Pardo J, Wu R, Carcangiu ML, Costa J and Tallini G: Ras mutations are associated with aggressive tumor phenotypes and poor prognosis in thyroid cancer. J Clin Oncol 21: 3226-3235, 2003.

15. Vasko V, Ferrand M, Di Cristofaro J, Carayon P, Henry JF and de Micco C: Specific pattern of RAS oncogene mutations in follicular thyroid tumors. J Clin Endocrinol Metab 88: 2745-2752, 2003.

16. Santarpia L, El-Naggar AK, Cote GJ, Myers JN and Sherman SI: Phosphatidylinositol 3-kinase/akt and ras/raf-mitogen-activated protein kinase pathway mutations in anaplastic thyroid cancer. J Clin Endocrinol Metab 93: 278-284, 2008.

17. Liu Z, Hou P, Ji M, Guan H, Studeman K, Jensen K, Vasko V, El-Naggar AK and Xing M: Highly prevalent genetic alterations in receptor tyrosine kinases and phosphatidylinositol 3-kinase/akt and mitogen-activated protein kinase pathways in anaplastic and follicular thyroid cancers. J Clin Endocrinol Metab 93: 3106-3116, 2008

18. Xing M: Genetic alterations in the phosphatidylinositol-3 kinase/Akt pathway in thyroid cancer. Thyroid 20: 697-706, 2010.

19. García-Rostán G, Costa AM, Pereira-Castro I, Salvatore G, Hernandez R, Hermsem MJ, Herrero A, Fusco A, Cameselle-Teijeiro J and Santoro M: Mutation of the PIK3CA gene in anaplastic thyroid cancer. Cancer Res 65: 10199-10207, 2005.

20. Wang ZG, Fukazawa T, Nishikawa T, et al: RAD001 offers a therapeutic intervention through inhibition of mTOR as a potential strategy for esophageal cancer. Oncol Rep 23: 1167-1172, 2010.

21. Xu DZ, Geng QR, Tian Y, et al: Activated mammalian target of rapamycin is a potential therapeutic target in gastric cancer. BMC Cancer 10: 536, 2010.

22. Faustino A, Couto JP, Pópulo H, Rocha AS, Pardal F, Cameselle-Teijeiro JM, Lopes JM, Sobrinho-Simões M and Soares P: mTOR pathway overactivation in BRAF mutated papillary thyroid carcinoma. J Clin Endocrinol Metab 97: E1139-E1149, 2012.

23. Jin N, Jiang T, Rosen DM, Nelkin BD and Ball DW: Dual inhibition of mitogen-activated protein kinase kinase and mammalian target of rapamycin in differentiated and anaplastic thyroid cancer. J Clin Endocrinol Metab 94: 4107-4112, 2009. 